\title{
$\dot{H}$ Global Clinical Research Journal
}

\section{Dimensionamento de enfermagem na nefrologia}

Nursing dimensioning in nephrology

Dimensionamiento de enfermería en nefrología

Gercilene Cristiane Silveira ${ }^{1}$

ORCID: 0000-0002-1642-6917

\author{
Adriane Lopes ${ }^{1 *}$ \\ ORCID: 0000-0001-7221-7012
}

${ }^{1}$ Faculdades Integradas de Jaú. São Paulo, Brasil.

*Autor correspondente: E-mail: lopesadriane98@gmail.com

\section{Resumo}

Objetivou-se comparar o dimensionamento de pessoal de enfermagem proposto por Raquel Rapone Gaidzinski, Fernanda Maria Tojeiro Fugulin e Valéria Castilho sobre "GERENCIAMENTO EM ENFERMAGEM" coordenado por Paulina Kurcgant com as diretrizes da Resolução da Diretoria Colegiada - RDC n.o 154, de 15 de junho de 2004. Trata de um estudo retrospectivo e descritivo do dimensionamento da equipe de enfermagem atuante no Serviço de Hemodiálise de um Hospital Universitário do Município de Campinas. A comparação entre os quadros de pessoal de enfermagem existente no Serviço de Hemodiálise do Hospital do Município de Campinas ao projetado, demonstrou carência de 9 enfermeiras, ausência de 4 auxiliares e excesso de 2 técnicos de enfermagem. A análise das variáveis intervenientes no processo de enfermagem apontou a necessidade de reorganização do processo de trabalho referente ao agrupamento de pacientes por complexidade sugerindo a aplicação, adaptação e validação de um instrumento na hemodiálise.

Descritores: Dimensionamento; Enfermagem; Hemodiálise; Gestão de Pessoas; Terapia Renal Substitutiva.

Como citar este artigo:

Silveira GC, Lopes A. Dimensionamento de enfermagem na nefrologia. Glob Clin Res. 2021;1(1):e5.

Editor Chefe: Caroliny dos Santos Guimarães da Fonseca

Editor Executivo: Kátia dos Santos Armada de Oliveira

Submissão: 18-03-2021

Aprovação: 30-03-2021 


\section{Abstract}

The aim was to compare the dimensioning of nursing staff proposed by Raquel Rapone Gaidzinski, Fernanda Maria Tojeiro Fugulin and Valéria Castilho on "MANAGEMENT IN NURSING" coordinated by Paulina Kurcgant with the guidelines of the Resolution of the Collegiate Board of Directors - RDC No. 154 of June $15^{\text {th }}, 2004$. This is a retrospective and descriptive study of the dimensioning of the nursing team working in the Hemodialysis Service of a University Hospital in the Municipality of Campinas. The comparison between the existing nursing staff in the Hemodialysis Service of the Hospital of the Municipality of Campinas and the projected one showed a shortage of 9 nurses, absence of 4 assistants and an excess of 2 nursing technicians. The analysis of the intervening variables in the nursing process indicated the need to reorganize the work process regarding the grouping of patients by complexity, suggesting the application, adaptation and validation of an instrument in hemodialysis.

Descriptors: Dimensioning; Nursing; Hemodialysis; People Management; Renal Sustainable Therapy.

\section{Resumén}

El objetivo fue comparar el dimensionamiento del personal de enfermería propuesto por Raquel Rapone Gaidzinski, Fernanda Maria Tojeiro Fugulin y Valéria Castilho sobre "GESTIÓN EN ENFERMERÍA" coordinado por Paulina Kurcgant con los lineamientos de la Resolución de la Junta Directiva Colegiada - RDC No. 154, de 15 de junio de 2004. Se trata de un estudio retrospectivo y descriptivo del dimensionamiento del equipo de enfermería que trabaja en el Servicio de Hemodiálisis de un Hospital Universitario de la Municipio de Campinas. La comparación entre el personal de enfermería existente en el Servicio de Hemodiálisis del Hospital del Municipio de Campinas con el proyectado, mostró una escasez de 9 enfermeras, ausencia de 4 auxiliares y un exceso de 2 técnicos de enfermería. El análisis de las variables intervinientes en el proceso de enfermería indicó la necesidad de reorganizar el proceso de trabajo en cuanto a la agrupación de pacientes por complejidad, sugiriendo la aplicación, adaptación y validación de un instrumento en hemodiálisis.

Descriptores: Dimensionamiento; Enfermería; Hemodiálisis; Gestión de Personas; Terapia de Reemplazo Renal.

\section{Introdução}

A Insuficiência Renal Crônica (IRC) vem se tornando um importante problema de saúde pública, pois a cada ano cresce de forma significativa o número de pessoas com esta doença e que requerem Terapia de Substituição Renal (TRS), não mostrando quaisquer sinais para fixar limites dentro das próximas duas décadas. ${ }^{1}$

Segundo a Sociedade Brasileira de Nefrologia (SBN) a Hipertensão e o Diabetes Melittus são responsáveis por $50 \%$ dos pacientes em diálise. Nos Estados Unidos a Hipertensão e o Diabetes Melittus são responsáveis por $3 / 4$ dos pacientes em diálise, o que pode ser parcialmente explicado pelo número de idosos dos países desenvolvidos. ${ }^{2}$

No Brasil vêm ocorrendo mudanças em termos de idade média da população. Atualmente o número estimado de idosos no Brasil é de 14,5 milhões e estima-se dobrar em 20 anos. Além do envelhecimento, o número de diabéticos e hipertensos sem diagnóstico e sem tratamento adequado são elevados. ${ }^{3}$

Estima-se que $25 \%$ da população Brasileira têm Hipertensão, ou seja, 26 milhões de pessoas. Apenas 15\% têm a pressão arterial devidamente controlada, dessa forma 85\% têm potencial para evoluir para DRC (Doença Renal Crônica). No Brasil e na América Latina 15\% dos pacientes em diálise são diabéticos e a taxa de mortalidade desses pacientes é sabidamente maior que a dos não diabéticos, decorrente do não desenvolvimento vascular destes pacientes no início da hemodiálise (processo arteriosclerótico). ${ }^{4,5}$

A alta incidência da Nefropatia diabética deve-se ao aumento da incidência de diabéticos tipo II na população em geral, decorrente da epidemia de obesidade. Estima-se que 20 a $45 \%$ dos pacientes diabéticos desenvolveram nefropatia diabética ao final de 10 - 15 anos de observação. Além disso, no Brasil, entre os indivíduos acima de 20 anos, 30 milhões estão acima do peso e desse total, mais de 10 milhões são considerados obesos que é outro fator de risco para a DRC. ${ }^{6}$

A SBN revelou que no Brasil há mais de 87.044 pacientes em programa de diálise em 2008, um crescimento de aproximadamente $9 \%$ nos últimos anos com uma taxa de incidência de 175 pmp e de prevalência de 383 pmp. Essas taxas de prevalência no Brasil são cerca de quatro vezes menores que a dos Estados Unidos e Japão, e duas vezes menores do que a da Itália, França e Alemanha. Isso se dá pelo baixo índice de diagnóstico precoce da DRC, acesso limitado a TRS, alta mortalidade dos pacientes diabéticos e hipertensos ainda na fase pré-dialítica. ${ }^{2}$

As modalidades terapêuticas são complexas, variadas e representam um problema de saúde de ampla magnitude e relevância, especialmente, quando se reconhece sua complexidade, seus riscos, sua diversidade de opções e o seu custo. As unidades de diálise oferecerem hemodiálise e diálise peritoneal de maneira efetiva, eficiente e segura adaptado à realidade local. Para que isto ocorra é imperativo o emprego racional de recursos. Desta maneira, 
os resultados passam a ser mensurados e avaliados, sendo que os beneficiados são dos cidadãos, dos profissionais responsáveis pela prestação da assistência e dos compradores dos serviços. ${ }^{7}$

Nessa perspectiva, este estudo objetiva comparar o dimensionamento de pessoal de enfermagem proposto por Raquel Rapone Gaidzinski, Fernanda Maria Tojeiro Fugulin e Valéria Castilho no Capítulo 10 de "GERENCIAMENTO EM ENFERMAGEM" coordenado por Paulina Kurcgant com as diretrizes da Resolução da Diretoria Colegiada - RDC número 154 , de 15 de junho de 2004, que estabelece o regulamento técnico para o funcionamento dos serviços de diálise. ${ }^{8-10}$

\section{Metodologia}

Trata de um estudo retrospectivo e descritivo do dimensionamento da equipe de enfermagem atuante no Serviço de Hemodiálise de um Hospital Universitário do município de Campinas e comparar o dimensionamento proposto por Raquel Rapone Gaidzinski com a Portaria do Ministério da Saúde Resolução da Diretoria Colegiada n.o 154 , de 15 de junho de 2004. ${ }^{8-10}$

A equipe multiprofissional é liderada por um médico nefrologista e uma enfermeira nefrologista, que são os responsáveis técnicos pelo serviço. A equipe é composta por 10 médicos (07 nefroadulto e 03 nefropediatra), 04 enfermeiros, 03 médicas residentes (02 nefroadulto e 01 nefropediatra), 02 enfermeiras residentes, 09 auxiliares de enfermagem, 15 técnicos de enfermagem, 02 nutricionistas (adulta e pediátrica), 01 assistente social e 01 psicóloga. As áreas de apoio (lavanderia, manutenção, central de materiais, laboratório de análises clínicas, diagnóstico por imagem entre outras) correspondem àquelas designadas para oferecer suporte a toda comunidade hospitalar.

O serviço de Nefrologia e Diálise de um Hospital Universitário do município de Campinas é uma unidade intra-hospitalar, pertencente ao hospital, com capacidade para 92 pacientes conveniados ao Sistema Único de Saúde e 12 pacientes com outros convênios e particulares. Conta com Ambulatório de Nefrologia, Acesso Vascular, Diálise Peritoneal, Pré-Transplante, Transplante, Nutrição, Psicologia e Serviço Social tanto adulto como criança.

A maior parte das admissões advém das unidades de internação e pronto-socorro, o que reflete alta incidência de pacientes com cateter venoso central como acesso vascular para hemodiálise e alta incidência de mortalidade, devido à gravidade com que estes pacientes são admitidos.

Os dados foram coletados pela pesquisadora, das escalas presenciais da equipe de enfermagem do Serviço de Hemodiálise de um Hospital Universitário do município de Campinas nos períodos de janeiro de 2007 à Dezembro de 2007 dos diferentes turnos de trabalho.

Após a obtenção das variáveis foram aplicados em equações de dimensionamento de pessoal de enfermagem segundo Gaidzinski e os resultados comparados a Resolução de Diretoria Colegiada n.o 154, de 15 de junho de 2004. ${ }^{8,9}$ Serão apresentados em números absolutos e percentuais, sob forma de discussão. $O$ estudo beneficiará a instituição ao fornecer subsídios para dimensionar o pessoal de enfermagem em hemodiálise.

Além disso, os resultados encontrados serão divulgados em congressos, eventos científicos e publicação em periódico científico.

Este estudo foi submetido à avaliação e aprovação do Comitê de Ética em Pesquisa da Pontifícia Universidade Católica de Campinas, protocolo n.․ 146/08 e o consentimento da diretoria do Hospital e Maternidade Celso Pierro de Campinas - SP.

\section{Resultados}

Considerando-se como ausências previstas os dias relativos às folgas e férias, ausências não previstas os dias relativos às faltas, as licenças e às suspensões no período de janeiro de 2007 a dezembro de 2007, o quadro a seguir destaca de forma detalhada os seguintes profissionais: enfermeiros, técnicos e auxiliares de enfermagem.

Quadro 1. Especificações das ausências previstas os dias relativos às folgas e férias, ausências não previstas os dias relativos às faltas, as licenças e às suspensões no período de janeiro de 2007 a dezembro de 2007. Jaú, SP, Brasil, 2008

\begin{tabular}{|c|c|c|c|c|}
\hline Categoria & Férias & Licenças & Faltas & Atestados \\
\hline Enfermeiro & 120 dias & 2 dias & 0 dias & 14 dias \\
\hline Técnico & 330 dias & 30 dias & dias & 78 dias \\
\hline Auxiliar & 333 dias & 10 dias & 24 dias & 79 dias \\
\hline Total & 783 dias & 42 dias & 24 dias & 171 dias \\
\hline
\end{tabular}

Aplicação da equação para dimensionar o pessoal de enfermagem

$$
A_{f} \%=\left(\frac{N_{f}}{D F-N_{f}}\right) \cdot 10
$$

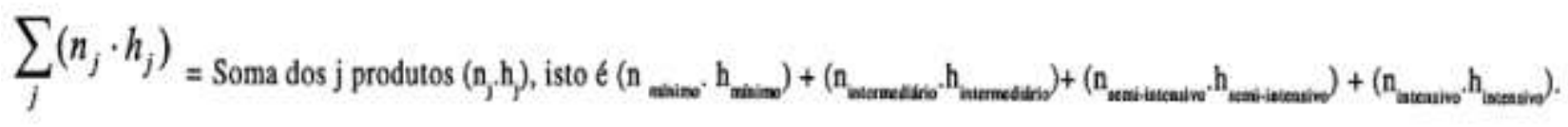




$$
q=\sum_{k} \frac{P_{k}}{100} \cdot \frac{\sum_{j}\left(n_{j} \cdot h_{j}\right)}{t \cdot p} \cdot\left[\left(1+\frac{e}{d-e}\right) \cdot\left(1+\frac{f}{D-f}\right) \cdot\left(1+\frac{v_{k}}{D-v_{k}}\right) \cdot\left(1+\frac{a_{k}}{D-a_{k}}\right)\right]
$$

Sendo:

q: quantidade total de pessoas de enfermagem;

Pk: proporção percentual da categoria k;

k:categoria profissional;

nj: quantidade média diária de pacientes que necessitem do tipo de cuidado;

j: tipo de cuidado requerido pelo paciente;

$\mathrm{h}$ : tempo médio de cuidado de cada paciente que necessita de cuidado;

t: tempo diário de trabalho de cada profissional dado em horas;

p: produtividade média (tempo diário de trabalho, dedicado ao cuidado do paciente);

e: número de folgas semanais que necessitam de cobertura; f: número de dias feriados por ano, não coincidentes com o domingo;

vk: número de dias de férias por ano, por categoria profissional;

ak: número médio de dias de ausência não prevista (absentismo) por categoria profissional;

$\mathrm{d}$ : dias de funcionamento da unidade (no caso 6 dias);

D: dias trabalhados no ano (no caso 365 dias).

O cálculo do pessoal de enfermagem foi realizado com inserção dos dados coletados em planilha, que facilita a operacionalização da equação, cedida pela Profa. Dra. Raquel R. Gaidzinski e as etapas deste cálculo foram armazenadas em um banco de dados. O dimensionamento do pessoal de enfermagem realizado segundo o modelo de Gaidzinski indicou um total de 13 enfermeiros, 13 técnicos e 13 auxiliares de enfermagem. A comparação entre os quadros de pessoal de enfermagem existente no Serviço de Hemodiálise do Hospital do Município de Campinas ao projetado, demonstrou carência de 09 enfermeiras, ausência de 04 auxiliares e excesso de 02 técnicos de enfermagem.

Os dados obtidos no Serviço de Nefrologia e Diálise do Hospital da PUC de Campinas foram aplicados na equação da Profa. Dra. Raquel R. Gaidzinsk e comparados com o quadro de dimensionamento de pessoal de enfermagem da Resolução da Diretoria Colegiada do Ministério da Saúde n.o 154:

1. Classificação dos pacientes: segundo Resolução COFEN/2004;

2. Índice de segurança técnico de $30 \%$;

3. Carga horária semanal: $\mathbf{3 6}$ horas;

4. Produtividade: $80 \%$;

5. Percentual de cada categoria profissional: mínimo/intermediário - 33\% a 37\% enfermeiros e o restante técnico e auxiliares de enfermagem, semi-intensivo $-42 \%$ a $46 \%$ de enfermeiros e o restante técnicos e auxiliares de enfermagem, intensivo - 52\% a $56 \%$ de enfermeiros e o restante técnicos e auxiliares de enfermagem.

O instrumento de classificação não foi aplicado pelo fato de que os instrumentos encontrados nunca foram testados em uma unidade de hemodiálise por não caracterizarem o perfil desta clientela.

Sendo que o agrupamento de pacientes por sistema de classificação da complexidade assistencial, além de melhorar a qualidade dos cuidados prestados, é viável também para alocar recursos humanos e materiais, diminuindo o custo dos cuidados de enfermagem.

Ao cliente crônico com idade superior a 60 anos, sem acompanhante, classificado com demanda de assistência intermediária ou semi-intensiva, deverá ser acrescido de 0,5 às horas de enfermagem especificadas no Artigo 4으 da Resolução 293/2004 e a distribuição percentual do total de profissionais de enfermagem, deve observar as seguintes proporções além da classificação dos pacientes na assistência mínima ou intermediária 33 a $37 \%$ de enfermeiros (mínimo de 06) e os demais, auxiliares e técnicos de enfermagem; na assistência semi-intensiva de 42 a $46 \%$ enfermeiros e os demais auxiliares e técnicos de enfermagem; na assistência intensiva 52 a $56 \%$ enfermeiros e os demais técnicos de enfermagem; A distribuição de profissionais por categoria deverá seguir o grupo de pacientes de maior prevalência.

Segundo a Resolução COFEN n.o 293/2004, a metodologia de cálculo de pessoal de enfermagem, para a clientela do serviço de diálise, se encaixa na assistência semiintensiva e/ou intensiva, levando em conta seu grau de prevalência sua assistência é semi-intensiva.

\section{Discussão}

O trabalho de enfermagem, enquanto parte do trabalho coletivo em saúde, apresentado anteriormente, realiza-se em diferentes instituições de saúde, sejam elas públicas ou privadas, caracterizadas pela internação ou não dos indivíduos. Nestas instituições, nas quais predomina o processo de produção em saúde centrado na finalidade de controlar as doenças em escala social e recuperar a força de trabalho incapacitada, o trabalho de enfermagem configurase como instrumento do trabalho em saúde, atuando sobre o mesmo objeto e reproduzindo o modelo clínico de organização desse processo, na medida em que se ocupa prioritariamente do cuidado individual dos corpos. ${ }^{11}$

Pesquisas $^{12-17}$ sobre dimensionamento de pessoal de enfermagem foram desenvolvidas, no entanto, nenhuma deles voltados aos pacientes em terapia dialítica, portanto 
este estudo evidencia a necessidade de adaptação de um instrumento para classificar a real complexidade do paciente portador de insuficiência renal terminal em programa de hemodiálise.

A previsão de pessoas para as atividades de enfermagem nos hospitais é um problema que se baseia, fundamentalmente, na determinação da categoria profissional que a instituição se dispõe a contratar e na definição das suas funções, o que, por sua vez, depende da filosofia e da política institucional, bem como do conceito que a direção tem da enfermagem. ${ }^{15}$

Exaustivos estudos destinados a calcular o pessoal de enfermagem têm sido desenvolvidos ao longo dos anos na tentativa de estabelecer um método que se ajuste às reais necessidades de diversos serviços. A contagem do número de leitos ocupados, a proporcionalidade de pessoal de enfermagem e a relação paciente e hora média de assistência de enfermagem têm sido experimentados como parâmetros para o dimensionamento de pessoal de enfermagem. ${ }^{8,17}$

A predominância do processo de trabalho gerencial do enfermeiro pode ser compreendida pelo número escasso de profissionais na composição da força de trabalho. ${ }^{18}$

Ressalta-se que, apesar do estudo realizado demonstrar que, pela aplicação do modelo de Gaidzinski, o quadro de pessoal de enfermagem da unidade de hemodiálise do Hospital da PUC de Campinas é defasado à necessidade de assistência aos pacientes em hemodiálise, embora enquadrado na portaria vigente RDC n.o 154. As causas atribuídas a esta percepção são: ausência de um instrumento adaptado aos pacientes em hemodiálise para que seja mensurada a sua real complexidade, enquanto estão sendo considerados semi-intensivos em alguns momentos ele se torna intensivo e em outros, intermediário; morosidade na substituição de licenças médicas e maternidade; precariedade de profissionais treinados nesta especialidade, aumentando o tempo de treinamento do mesmo.

O estudo permitiu concluir que o quadro existente na clínica respeita e até supera, discretamente, o preconizado pela Resolução COFEN n. ${ }^{\circ}$ 293/2004.

\section{Considerações Finais}

A comparação entre o quadro de funcionários existente no Serviço de Hemodiálise do Hospital da PUC de Campinas e o projetado, demonstrou carência de 09 enfermeiros, 04 auxiliares de enfermagem e excesso de 02 técnicos de enfermagem.

Análise das variáveis intervenientes no processo de enfermagem apontou a necessidade de reorganização do processo de trabalho referente ao agrupamento de pacientes por complexidade sugerindo a aplicação, adaptação e validação de um instrumento na hemodiálise, onde seria demonstrado o tempo destinado à prestação da assistência de enfermagem aos pacientes em hemodiálise.

No entanto, a equipe de enfermagem, frequentemente, refere que existe grande sobrecarga de trabalho, tendo a percepção de que o quadro de pessoal da unidade é menor do que o necessário. Eles são frequentemente submetidos a inapropriado trabalho contínuo de origem física e psicológica, causando-lhes desordens fisiológicas. Essa talvez possa ser uma das justificativas para o alto índice de absenteísmo e licença não prevista.

\section{Referências}

1. Ministério da Saúde (BR). Insuficiência renal crônica. 2015. Acesso em 18/12/2020. Disponível em: https://bvsms.saude.gov.br/dicas-emsaude/2083-insuficiencia-renal-cronica

2. Sociedade Brasileira de Nefrologia. SBN Censo 2017. Acesso em 18/12/2020. Disponível em: https://www.sbn.org.br/fileadmin/user_upload/informa/sbninforma114-2.pdf

3. Miranda GMD, Mendes ACG, Silva ALD. O envelhecimento populacional brasileiro: desafios e consequências sociais atuais e futuras. Rev. Bras. Geriatr. Gerontol., Rio de Janeiro, 2016; 19(3):507-519. https://doi.org/10.1590/1809-98232016019.150140

4. Francisco PMSB, Segri NJ, Borim FSA, Malta DC. Prevalência simultânea de hipertensão e diabetes em idosos brasileiros: desigualdades individuais e contextuais. Ciênc. saúde coletiva. 2018; 23(11). http://dx.doi.org/10.1590/1413-812320182311.29662016

5. Sociedade Brasileira de Geriatria e Gerontologia. . OMS divulga metas para 2019; desafios impactam a vida de idosos. 2019. Acesso em 18/12/2020. Disponível em: https://sbgg.org.br/oms-divulga-metas-para-2019-desafios-impactam-a-vida-de-idosos/

6. Sociedade Brasileira de Diabete. Diretrizes da sociedade brasileira de diabetes 2019-2020. Acesso em 18/12/2020. Disponível em: http://www.saude.ba.gov.br/wp-content/uploads/2020/02/Diretrizes-Sociedade-Brasileira-de-Diabetes-2019-2020.pdf

7. Mendes ML, Alves CA, Bucuvic EM, Dias DB, Ponce D. . Diálise peritoneal como primeira opção de tratamento dialítico de início não planejado. J. Bras. Nefrol. 2017; 39(4). http://dx.doi.org/10.5935/0101-2800.20170077

8. Brasil. Resolução - RDC no 154, de 15 de junho de 2004. Estabelece o Regulamento Técnico para o funcionamento dos Serviços de Diálise Acesso em 18/12/2020. Disponível em: http://bvsms.saude.gov.br/bvs/saudelegis/anvisa/2004/rdc0154_15_06_2004_rep.html

9. Gaidzinski RR. Dimensionamento do pessoal de enfermagem segundo a percepção de enfermeiros que vivenciam essa prática. São Paulo, 1994. 259p. Tese (Doutorado) - Escola de Enfermagem, Universidade de São Paulo.

10. Kurcgant P. Gerenciamento em enfermagem. Rio de Janeiro, Guanabara Koogan, 2005. Cap.10. p. 125-137.

11. Conselho Federal de Enfermagem (COFEN). Resolução COFEN n.o 543/2017. Acesso em 18/12/2020. Disponível em: http://www.cofen.gov.br/resolucao-cofen-5432017_51440.html

12. Silva RGM, Nascimento VF, Bertucci AAS, Benicio AC, Ferreira DS, Lopes CCC. Análise reflexiva sobre a importância do Dimensionamento de Pessoal de Enfermagem como ferramenta gerencial. 2016; 15(4).

13. Lorenzini E, Deckmann LR, Silva EF. Dimensionamento de pessoal de enfermagem em centro obstétrico. Rev Enferm UFSM 2015 Out./Dez.;5(3):661-668. Doi: 10.5902/2179769215038 
14. Araujo MT, Velloso ISC, Queiroz CF, Henriques AVB. Dimensionamento de pessoal de enfermagem de uma unidade de clínica médica. 2016; 6 (2). DOI: https://doi.org/10.19175/recom.v6i2.971

15. Silva LC da, Oliveira DAL, Santos ABR, Barbosa LMS, Araújo LG, Barboza MTV, et al. Dimensionamento de pessoal e sua interferência na qualidade do cuidado. Rev enferm UFPE on line., Recife, 2019; 13(1):491-8.

16. Marcolongo APF. Follow-up of the audit in the implementation of the checklist at the medical records conference in a philanthropic institution. Glob Acad Nurs. 2020;1(2):e24.https://dx.doi.org/10.5935/2675-5602.20200024

17. Mendes RNC, Carmo AFS, Salum RDL et al. Dimensionamento de pessoal: avaliação da enfermagem em unidades de terapia intensiva obstétrica e pediátrica mista. R. pesq.:cuid. fundam. online 2013. abr./jun. 5(2):3706-16. DOI: 10.9789/21755361.2013v5n2p3706

18. Pereira BSL, Pereira SRM, Mesquita AMF et al. Aplicação do Nursing Activities Score (NAS) em uma Unidade de Terapia Intensiva (UTI). R. pesq.: cuid. fundam. online 2020 jan/dez 12: 79-87. DOI: 10.9789/2175-5361.rpcfo.v12.7052

19. Silva MCN, Machado MH. Sistema de Saúde e Trabalho: desafios para a Enfermagem no Brasil. Ciência \& Saúde Coletiva, $2020 ; 25(1): 7-13$. https://doi.org/10.1590/1413-81232020251.27572019

20. Schneider F. Práticas Avançadas de Enfermagem: conceitos e estratégias na implantação. Glob Acad Nurs. 2020;1(2):e11.https://dx.doi.org/10.5935/2675-5602.20200011 